Author(s):

Submitted to:
Applications of Mathematical Analysis of Nonlinear Physical Systems
H. Frauenfelder, T CNLS

P. Hagan, ClC-3

J. Sobehart, CIC-19

Tetsuji Ueda, Northwestern University

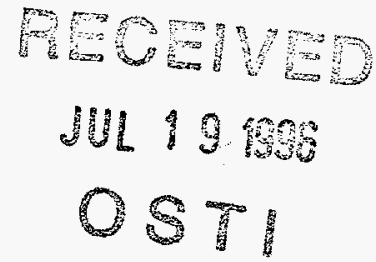

DOE Office of Scientific and Technical Information (OSTI)

\section{Los Alamos}

NATIONAL LABORATORY

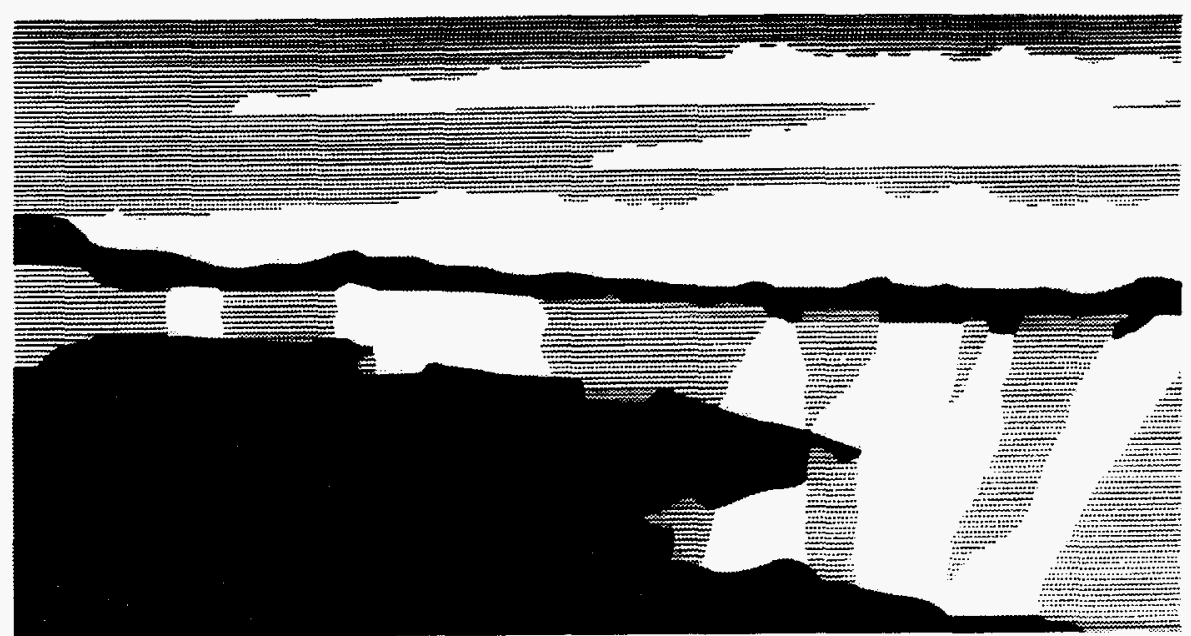

Los Alamos Nationa! Laboratory, an affirmative action/equal opportunity employer, is operated by the University of California for the U.S. Department of Energy under contract W-7405-ENG-36. By acceptance of this article, the publisher recognizes that the U.S. Government retains a nonexclusive, royaltyfree license to publish or reproduce the published form of this contribution, or to allow others to do so, for U.S. Government purposes. The Los Alamos

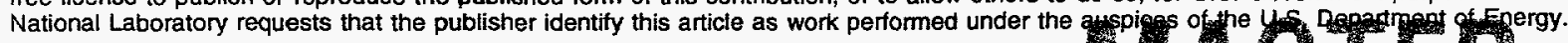




\section{DISCLAIMER}

Portions of this document may be illegible in electronic image products. Images are produced from the best available original document. 


\title{
Applications of Mathematical Analysis of Nonlinear Physical Systems
}

\author{
Hans Frauenfelder*, Patrick Hagan, Jorge Sobehart, \\ and Tetsuji Ueda (Northwestern University)
}

\begin{abstract}
This is the final report of a three-year, Laboratory-Directed Research and Development (LDRD) project at the Los Alamos National Laboratory (LANL). In this project we have sought to acquire the basic theoretical and computational capabilities needed to advance our understanding of the physical sciences. We concentrated on physical systems governed by transport equations. Transport equations were chosen because they govern many critical technologies. They govern the flow of carriers in semiconductors, the dynamics of nuclear reactors, the transport of radiation in fusion experiments, and even the probability densities of stochastic equations. Besides being important in its own right, this research serves as an important paradigm for using computers to solve other key scientific problems. Transport problems are often characterized by mean free paths that are short compared to the other critical length scales of the system. We exploit this feature to both simplify the solution of transport equations and to gain insights into the results. To explore industrial applications of the science developed in this project, a workshop was sponsored highlighting eight to ten problems from industry. Promising research avenues were uncovered that might lead to potential collaborations with industry.
\end{abstract}

\section{Background and Research Objectives}

Transport (Boltzmann) equations express how particle densities $f(t, x, k)$ evolve in $\mathrm{x}-\mathrm{k}$ phase space, where $\mathrm{k}$ is the particle momentum. Using Monte Carlo (MC) methods to directly solve the Boltzmann equation is often exceedingly slow due to its high dimensionality (time, three space, and three momentum dimensions). For example, MC programs such as DAMOCLES at IBM, are usually limited to steady-state simulations in

*Principal investigator, e-mail: frauenfelder@lanl.gov 
two space dimensions; even so, it often takes a supercomputer several days to simulate a single semiconductor device using DAMOCLES. We conclude that using MC methods to perform transient, three-dimensional simulations on a routine basis will not be feasible in the foreseeable future.

There are other ways to model and predict transport phenomena. These include much simpler reduced-dimensionality models, such as drift-diffusion (variable mobility) models and hydrodynamic models. These approximate models can be solved readily on present-day computers. However, their predictions are rather unreliable outside very restricted domains-of-validity.

Transport problems are often characterized by mean free paths that are short compared to the other critical length scales of the system, and this feature can be exploited to both simplify the solution of transport equations and to gain insights into the results. We therefore consider systems with small mean free paths, systematically exploiting this characteristic to develop a hybrid analytical/numerical approach to solving - and understanding - these systems. An important application is to the physics of semiconductors, where we use this approach to develop efficient new models for designing advanced semiconducting devices.

Many novel electronic devices are being developed that exploit electron nonlocality, including tunneling devices, fine-scale heterostructures, and other nano-devices. Designing such devices is often difficult because state-of-the-art transport equations can only account for electron non-locality in a very ad hoc manner. Our objective is to derive a transport equation from first principles which is analogous to the semiclassical equations, but which correctly accounts for non-locality. In addition, when electrons pass through certain symmetry points, such as the $\mathrm{X}$ point in $\mathrm{Si}$, quantum resonance occurs. We analyze these resonances to determine what phenomena it causes, and how these phenomena can be exploited.

\section{Importance to LANL's Science and Technology Base and National R\&D Needs}

Phenomena governed by transport equations are fundamental to many areas of science and engineering that are critical to the nation's technological progress. Transport calculations arise routinely in microelectronic design, health physics, environmental safety, controlled fusion research, inertial-confinement research, radiation shielding, various forms of well logging, radiation therapy planning, nuclear reactor design and analysis, nuclear weapons design, ion implantation, and many other areas. General improvement in our 
theoretical and computational understanding of transport phenomena can impact a wide spectrum of scientific and engineering communities. Transport phenomena fit naturally with LANL's inherent strength in advanced computational methods, physics, and mathematical analysis and modeling.

\section{Scientific Approach and Results}

In this project we systematically develop new reduced-dimensionality models that very accurately simulate particle transport, even in regimes corresponding to strong and rapidly varying forces. To do this, we analyze the Boltzmann equation using singular perturbation techniques to exploit the shortness of the mean free path. This analysis determines the physical regimes possible for the transport system, and it enables us to derive the unique reduced-dimensionality equation which is asymptotically accurate in each regime.

In transport problems there are very thin 'kinetic' boundary layers next to all spatial boundaries and material interfaces. Reduced dimensionality equations represent an 'outer solution' to the Boltzmann equation, and are not valid in such layers. Instead, these boundary layers must be resolved and matched to the reduced equations. This matching procedure then provides the effective boundary conditions for the reduced equations. Together, the reduced dimensionality equation and these effective boundary conditions form the complete asymptotically-accurate model for the transport problem.

\subsection{Carrier Transport in Semiconductors}

Our research pertains to general transport equations. However, for clarity, we prefer to express it in terms of a specific application, namely carrier transport in semiconductors. Using singular perturbation techniques we have discovered that there are two distinct limits (physical regimes) possible for semiconductors, the pseudohomogenous and strong-gradient regimes, depending on how rapidly the internal electric fields change with position.

Analysis of the pseudo-homogenous regime. In this regime, our analysis shows that carrier densities can be predicted (to within a transcendentally small error) by an equation that is very similar to a variable mobility (drift-diffusion) model. The only difference is that it contains a completely unexpected dispersive term. Since the new model simulates the Boltzmann equation very accurately, it has allowed us to resolve a longstanding controversy about carrier diffusivities. Theorists claim the diffusivity must be proportional to the lattice temperature (to get the steady state right) and experimentalists 
claim that it is proportional to the electron temperature, which is often twenty times larger. The diffusivity is proportional to the lattice temperature as the theorist claim, but in the experiments performed the new dispersive term makes it appear that the diffusivity is proportional to the electron temperature. In these experiments (and in some other transient situations) the new dispersion term is much more important than the classical diffusion term.

Engineers commonly predict device performance from variable mobility models by directly incorporating the experimentally measured diffusivity in the diffusion term. Our new model shows that this is treacherous; if the experimental diffusivity is not divided into its diffusive and dispersive components, then the predicted steady state for the device will generally be far from its true state.

The only material properties needed by the new model are the semiconductor's drift velocity and 'experimental diffusivity' as functions of the electric field strength. This is data that is readily available experimentally, and widely reported in the literature.

Analysis of the strong gradient regime. For this regime we initially derived reduced models by exploiting the timescale disparity between elastic (energy preserving) and inelastic scattering. This proved inadequate because the timescales are not sufficiently different for high energy electrons. However, the main scattering mechanisms (acoustic, optical, polar scattering, and impurity scattering) can only transfer at most $0.06 \mathrm{eV}$ of energy per event. So we are now analyzing the Boltzmann equation exploiting the fact that only a small amount of energy can be transferred per scattering event. Carrier-carrier scattering and impact ionization, which occur much less frequently, need to be handled separately. Unlike the drift-diffusion-dispersion model, this analysis should yield a reduced dimensionality model that directly incorporates 'diffusion' of the carriers in band energy.

Quantum non-locality. Recently we used dispersive wave techniques to derive the semiclassical equations (plus quantum corrections) from the Schrödinger equation. We should be able to use this technique to analyze the Wigner-Boltzmann formulation of quantum mechanics within a crystal. This will lead to equations analogous to the semiclassical equations, except that they will explicitly contain electron non-locality.

Semiconductor Devices. In this project we re-analyze the basic physics of semiconductors in the physical regimes corresponding to ultra-small, ultra-fast devices; use singular perturbation techniques to derive reduced dimensionality equations that accurately describe the semiconductor's electronic properties in these regimes; and validate the resulting theoretical models against Monte Carlo simulations and experimental data. From this we gain the capability of simulating the time-dependent operation of ultra-small ultra- 
fast devices in three spatial dimensions. This capability should greatly aid the design of new devices.

Systematic analysis of the Boltzmann equation shows that there are four asymptotic regimes possible for a semiconductor device, depending on the strength of its internal electric fields and on how rapidly the fields change with position. The key asymptotic regimes for advanced devices are the

(i) strong-scattering, strong-force regime,

(ii) weak-scattering, weak-force regime, and

(iii) weak-scattering, strong-force regime.

We believe these regimes will include most devices with feature sizes between roughly $0.07 \mu \mathrm{m}$ and $1.0 \mu \mathrm{m}$; future generations of advanced devices will almost certainly operate in this regime. Our first objective is to derive the reduced-dimensionality model that accurately simulates the full Boltzmann equation in each of these regimes.

In the strong-scattering, strong-force regime, we have derived the reduced dimensionality model that simulates the Boltzmann equation. This model (called the highfield semiconductor equations) does not yet account for boundary layer phenomena, such as velocity overshoot. This unduly limits its accuracy in simulating most advanced devices. We are currently developing a general method for resolving and matching boundary layers in kinetic equations. Once developed, we intend to apply this methodology to semiconductors, and then use it to develop a variant of the high-field equations that automatically handle the boundary layers.

We have also derived the reduced dimensionality model for the weak-scattering, weak-force regime, mainly due to the efforts of our collaborators at IBM. This model (known as the energy group drift-diffusion equations) has been compared to Monte Carlo simulations of the full Boltzmann equations. We discovered that it is quite accurate within its domain of validity.

In essence, the weak-scattering, strong-force regime combines both the previous regimes. If we can successfully develop an accurate model for the strong-scattering, strong-force regime, we foresee no difficulties in extending this analysis to the weakscattering limit.

Many novel electronic devices are being developed that exploit electron non-locality and other quantum effects. Designing these devices is impeded because state-of-the-art transport equations account for electron non-locality in a very ad hoc manner. To resolve this difficulty, we adapted Wigner's approach for electrons in free space to electrons in semiconductors by using multi-space expansion techniques. This enabled us to systematically derive the quantum corrections to the Boltzmann equation. Among these 
corrections are dispersion terms that determine how the electron probability density spreads out from the semiclassical trajectories; i.e., that govern electron non-locality. This extends the reach of the Boltzmann equation to situations where non-locality is important, such as tunneling devices, heterostructures, and nano devices.

Another focus of study was the development of a variational principle for upper bounds on the largest possible time-averaged convective heat flux, derived directly from the Boussinesq equations of motion. When supplied with appropriate test background fields satisfying a spectral constraint (reminiscent of an energy stability condition), the variational formulation produces rigorous upper bounds on the Nussalt $(\mathrm{N})$ number as a function of the Rayleigh number (R). Euler-Lagrange equations for the optimal background flow and temperature fields have been derived. In a simplified (but still rigorous) formulation where incompressibility is not fully exploited, we proved a large Rayleigh number bound $\mathrm{N} \leq \sqrt{\mathrm{R}} / 36-1$ for vertical heat connection between parallel plates.

\subsection{Exploration of Industrial Applications}

To explore the application of our work to industrial problems we hosted a workshop with several participants from the industrial community. Industrial problems rarely occur as a set of equations; more often they occur as a description of a phenomena that is troubling an industry or that the industry wishes to achieve, sometimes accompanied by experimental data. For a typical industrial problem (if there is such a thing), the attention is first directed at defining the problem, i.e., determining what needs to be learned to carry out the industrial mission. Effort is then directed at determining precisely which physical mechanisms are involved, what roles the different mechanisms play, and how these mechanisms interact together. Finally, effort is directed at deriving the mathematical equations that accurately describe these mechanisms. Only then can we apply our full range of analytical and numerical methods, developing an intuitive framework of how the system works, as well as a quantitative understanding of the system. The workshop participants then gather in working groups to formulate a rational approach to solving the problems.

The workshop studied the following six problems:

1. Determining low frequency index data from refracted/diffracted rays (Corning, Inc.).

2. Gas jet cleaning of filters (Pall Corp.).

3. Cracks in laser and arc welds (Rockwell Corp.).

4. Large gas release events from "dry" waste tanks (Pacific Northwest Lab.).

5. Reducing the cost of Monte Carlo analysis of well logging data (Chevron Petroleum).

6. Uncertainty in the solution of porous media flow models (Golder Associates). 
In all cases a number of approaches with high probability of success were suggested. This workshop therefore successfully identified several areas where the results of our scientific work might be applied to industrial problems. The workshop represented a useful culmination of the technical effort and projected potential collaborative effort between LANL and industrial researchers.

\section{Publications}

1. Hagan, P. S.; Cox, R. W. and Wagner, B. A. "Derivation of the High-Field Semiconductor Equations," in Semiconductors Part II, Coughlan et al. eds., IMA 59, pp.159-184 (1994).

2. Ueda, Tetsuji, "Semiclassical Equations for Electron Transport in Short Semiconductor Devices," Los Alamos document LAUR-93-0508, for presentation at the 1993 Arizona Days Workshop (1993).

3. Sobehart, Jorge R. "A Monte Carlo Simulation Approach to Semiconductor Device Modeling," Los Alamos document LAUR-93-3845, for presentation at the International Workshop on Numerical Modeling of Processes and Devices for Integrated Circuits: NUPAD V (1994).

4. Sobehart, Jorge and Hagan, Patrick, "Determination of Velocity Overshoot in Semiconductor Devices via Half-Range Resolution of the Boundary Layer," Los Alamos document LAUR-94-4056, for presentation at The Third International Congress on Industrial and Applied Mathematics, July 2-7, 1995, at Hamburg, Germany.

5. Sobehart, Jorge, "The High-Field Semiconductor Equations," Los Alamos document LAUR-94-4055, for presentation at The Third International Congress on Industrial and Applied Mathematics, July 2-7, 1995, at Hamburg, Germany.

\section{DISCLAIMER}

This report was prepared as an account of work sponsored by an agency of the United States Government. Neither the United States Government nor any agency thereof, nor any of their employees, makes any warranty, express or implied, or assumes any legal liability or responsibility for the accuracy, completeness, or usefulness of any information, apparatus, product, or process disclosed, or represents that its use would not infringe privately owned rights. Reference herein to any specific commercial product, process, or service by trade name, trademark, manufacturer, or otherwise does not necessarily constitute or imply its endorsement, recommendation, or favoring by the United States Government or any agency thereof. The views and opinions of authors expressed herein do not necessarily state or reflect those of the United States Government or any agency thereof. 